Archives de sciences sociales des religions

147 | juillet-septembre 2009

Traduire l'intraduisible

\title{
Le Sūtra de l'Estrade dans la Corée contemporaine
}

\section{Bernard Senécal}

\section{OpenEdition}

Journals

Édition électronique

URL : http://journals.openedition.org/assr/21451

DOI : $10.4000 /$ assr. 21451

ISSN : $1777-5825$

Éditeur

Éditions de l'EHESS

Édition imprimée

Date de publication : 1 octobre 2009

Pagination : 209-227

ISBN : 978-2-7132-2217-7

ISSN : 0335-5985

Référence électronique

Bernard Senécal, « Le Sūtra de l'Estrade dans la Corée contemporaine », Archives de sciences sociales des religions [En ligne], 147 | juillet-septembre 2009, mis en ligne le 01 octobre 2012, consulté le 01 mai 2019. URL : http://journals.openedition.org/assr/21451; DOI : 10.4000/assr.21451 


\section{Bernard Senécal}

\section{Le Sütra de l'Estrade dans la Corée contemporaine}

C'est en novembre 1987, à quelques mois de la tenue des jeux olympiques de Séoul, durant l'été 1988, lesquels avaient été précédés de la transition politique de la Corée du Sud d'une dictature militaire à un régime démocratique qui s'est maintenu jusqu'à aujourd'hui, qu'a été publiée la première traduction en langue coréenne de la version de Dunhuang du Sūtra de l'Estrade ${ }^{1}$. Son auteur n'était autre qu'un bonze déjà fort connu du grand public coréen, le Vénérable Sŏngch'ŏl ${ }^{2}$ (1912-1993), Patriarche du Chogyejong ${ }^{3}$, de loin le plus puissant ordre monastique bouddhiste de toute la Corée du Sud. Cette première traduction de la version la plus ancienne qui soit connue du célèbre sūtra chinois - dont la rédaction est attribuée par la tradition au $\mathrm{VI}^{\mathrm{e}}$ Patriarche Huineng en personne, la figure emblématique par excellence de l'école méditative extrême-orientale ${ }^{4}$ a suscité la production de plusieurs autres traductions du même manuscrit, dont les contenus vont du quasi plagiat de Sŏngch'ŏl à la complète remise en cause des options doctrinales de ce dernier.

1. L'oasis de Dunhuang constitue l'une des principales étapes traditionnelles de la route de la soie et se trouve située à l'extrémité est du désert de Gobi. Au début du siècle, des dizaines de milliers de manuscrits datés $d u I^{\mathrm{e}}$ au $\mathrm{X}^{\mathrm{e}}$ siècle y ont été retrouvés dont la découverte est d'une importance historique comparable à celle des manuscrits de la mer Morte. La version de Dunhuang du Sütra de l'Estrade est le manuscrit Stein 5475 ; elle correspond au volume 2007 du tome 48 de l'Édition Taishō du canon bouddhique sino-japonais; c'est la copie, réalisée entre 830 et 860 , d'un manuscrit plus ancien. Il en existe trois traductions en français; leurs coordonnées se trouvent en bibliographie.

2. Nom signifiant «celui qui a complètement réalisé sa nature (de Buddha) ». Cet article utilise le système McCune-Reischauer pour la romanisation des termes coréens ( $\breve{o}$ se prononce comme l'«a » de l'anglais «what»; "ch » comme le «tch» de Tchad; l'apostrophe marque, suivant les cas, l'aspiration du son qui la précède ou la coupure syllabique; $\breve{u}$ est un son produit par le fond de la gorge, à mi-chemin entre le «e » et le «eu»français; " $u$ » se prononce « ou », « oe », « wè » et « $j$ ", « dj »).

3. "Chogye " est un surnom donné au VI ${ }^{\mathrm{e}}$ Patriarche Huineng en raison du nom de la montagne de la province chinoise de Guandong où se trouve le monastère du même nom où il a longtemps résidé.

4. École qui comprend les courants " Chan » chinois, "Thiê `n " vietnamien, «Zen » japonais et «Sŏn » coréen (ces quatre noms sont tous des translittérations du sanskrit dhyāna, signifiant "méditation ", et dont la dernière syllabe est tombée), chacun de ces courants étant lui-même subdivisé en plusieurs autres. 
On peut ainsi s'interroger sur la signification de l'acte de traduction de la version de Dunhuang du Sütra de l'Estrade dans la Corée contemporaine : acte d'autant plus paradoxal qu'il tend à absolutiser un texte dont le génie est censé être de se refuser à ce genre de tentative. Selon toute vraisemblance, loin d'être le résultat d'une coïncidence fortuite, ce phénomène s'inscrit dans un contexte formé par une constellation de données historiques, sociopolitiques, économiques, religieuses. Dans cette perspective, on tentera de définir les faits permettant de mieux comprendre la production ininterrompue en Corée du Sud, et depuis 1987, d'un nombre significatif de traductions du Sütra de l'Estrade dans sa version de Dunhuang ${ }^{5}$.

\section{Brève histoire du bouddhisme coréen}

Introduit de Chine dans le nord de la péninsule coréenne vers 371, le bouddhisme du pays du Matin Calme n'a de cesse de mettre en avant, avec une réelle fierté, ses seize siècles d'histoire. Il n'en a pas moins connu, après pratiquement un millénaire de gloire, couvrant une partie de l'époque des Trois Royaumes ${ }^{6}$ (?-668), puis toute la durée de celles du Grand Silla (668-935) et de Koryŏ (9181392), une longue période de décadence, laquelle a commencé dès la naissance de la dynastie confucéenne de Chosŏn (1892-1910) et s'est achevée à la fin de la colonisation japonaise (1910-1945). Après la fin de la Guerre de Corée (19501953 ) et plus encore à partir du début de la présidence du général Pak Chŏng Hŭi, en 1960, ce bouddhisme a commencé à renaître de ses cendres, non sans avoir à affronter de graves difficultés. Car il n'est pas exagéré de dire qu'il est ressorti exsangue d'une période d'une durée de pratiquement six siècles pendant lesquels il a été, sinon brutalement persécuté, systématiquement asservi et utilisé par le pouvoir politique.

À la naissance de la dynastie Chosŏn, le bouddhisme péninsulaire perd soudainement et complètement le soutien politique sans partage dont il avait pu jouir à titre de religion d'État pendant les dix siècles précédents. Car la nouvelle dynastie met presque aussitôt en place l'ŏkpul sungyu chŏngch'aek : politique systématique d'oppression du bouddhisme et de promotion du néoconfucianisme du Chinois Zhuxi (1130-200) ${ }^{7}$. Celle-ci impose non seulement la fusion de toutes les écoles existantes en une seule - que leur identité soit fondée sur la scolastique ${ }^{8}$ ou la pratique de la méditation ${ }^{9}$ - mais encore la suppression intégrale du droit de cité des bonzes. Cette politique aura très vite pour conséquence

5. Cette recherche n'entend cependant pas, pour autant, réduire ledit phénomène aux données fournies par les sciences sociales.

6. Koguryŏ (?-668), Paekche (?-664) et Silla (?-935).

7. Il s'agit d'une doctrine extrêmement intolérante qui persécutera non seulement les bouddhistes mais encore les chamanes et, au XIX ${ }^{\mathrm{e}}$ siècle, les chrétiens.

8. Étude des textes ou kyo.

9. Appelée sŏn. 
la disparition complète du bouddhisme urbain et la naissance d'un bouddhisme qui, ayant trouvé refuge au cœur des montagnes ${ }^{10}$, cherchera à survivre en se centrant sur ce qui lui demeurait possible : la pratique de la méditation ${ }^{11}$. Vers la fin de cette dynastie, ce bouddhisme des montagnes aura atteint un état de déliquescence tel qu'il avait définitivement perdu la trace de ses lignées de transmission du Dharma ${ }^{12}$.

La restauration du droit de cité des bonzes coréens en 1895 permettait au bouddhisme du pays du Matin Calme d'espérer l'avènement d'une nouvelle ère de prospérité. Il n'en fut rien, bien au contraire. Car la puissance japonaise - qui avait mis un pied dans la péninsule dès la fin du XIX ${ }^{\mathrm{e}}$ siècle après un appel au secours de la royauté coréenne confrontée à de redoutables soulèvements paysans - allait se servir systématiquement du bouddhisme pour contrer l'influence des confucéens de la dynastie précédente et affirmer son emprise sur la Corée ; celle-ci allait devenir, à partir de 1910, l'une des colonies de son empire naissant. Les gouverneurs japonais n'eurent guère de mal à soumettre un bouddhisme leurré par la perspective d'une résurgence et, dans son ensemble, beaucoup trop affaibli pour pouvoir faire preuve d'esprit critique et résister. Leur tâche fut d'autant plus facile qu'ils s'étaient arrogés le pouvoir de nommer les abbés de tous les grands monastères, sous le contrôle desquels se trouvaient directement placés tous les autres temples et ermitages de la péninsule. De plus, afin de stabiliser et contrôler plus efficacement une population monastique essentiellement célibataire et très mobile, les gouverneurs exhortèrent fermement les bonzes à prendre épouse et fonder foyer. Pour être bien comprises, ces mesures adoptées par le régime colonial nippon à l'égard du bouddhisme coréen doivent être resituées dans le contexte d'une politique de japonisation intégrale de toute la Corée, extrêmement brutale et systématique, qui alla jusqu'à interdire l'enseignement et l'usage de la langue coréenne dans le système d'éducation.

Si la capitulation du Japon, en 1945, libère la Corée du joug de l'Empire du Soleil Levant, elle provoque également le partage de la péninsule en un Nord communiste, dominé par l'URSS, et un Sud capitaliste sous l'égide des forces alliées $^{13}$. Bien qu'elle ait fait quelque trois millions de morts et laissé tout le pays dans un état de complète dévastation, la Guerre de Corée n'aura pratiquement rien changé à cette situation qui perdure jusqu'à aujourd'hui. Tandis qu'en

10. Plus de $75 \%$ de la surface de la péninsule coréenne sont couverts de montagnes.

11. Dans la Corée du Sud contemporaine, la répartition géographique des temples et monastères demeure toujours dominée par les conséquences de cette politique.

12. La transmission de maître à disciple de l'enseignement bouddhique prétend remonter jusqu'au Buddha Śākyamuni en personne ; son intégrité est essentielle au maintien de l'identité des écoles méditatives.

13. Avant la fin de la Seconde Guerre mondiale, et sans avoir consulté les Coréens, les États-Unis et l'URSS s'étaient mis d'accord pour partager la péninsule au niveau du $38^{\mathrm{e}}$ parallèle. 
Corée du Nord le culte du président Kim Ilsŏng ${ }^{14}$ (1912-1994) commence à s'imposer comme religion d'État et interdit strictement toute autre forme de pratique religieuse (Hitchens, 2007 : 297-300), la Corée du Sud adopte au contraire une politique consistant à favoriser le développement de toutes les religions.

C'est à cette époque que correspond la présidence de Sungman Rhee (19481960), un méthodiste convaincu dont l'attitude à l'égard du bouddhisme sera pour le moins ambiguë. En effet, tout en veillant à la construction de lieux de culte protestants jusque dans les moindres petits villages de toute la partie sud de la péninsule, il promulgua, en 1954, l'édit Yusin ( « renouveau »), qui ordonnait la purgation du bouddhisme sud-coréen de tous ses éléments nippons. Cet ordre eut pour conséquence l'expulsion des monastères et temples de tous les bonzes mariés et de leurs familles. Les bonzes demeurés célibataires étant largement minoritaires, ils durent avoir recours à l'embauche d'hommes de main. L'administration du président Rhee laissa les événements suivre leur cours sans intervenir. Cette violente purge se termina sous la dictature militaire, à la fin des années soixante, par un schisme entre les bonzes célibataires qui fondèrent l'ordre Chogye et les bonzes mariés qui fondèrent l'ordre T'aego.

Pendant l'époque qui suit, la Corée du Sud va être successivement dirigée par trois militaires, tous originaires de la ville de Taegu dans la province du Kyŏngsang du Nord. Dès 1960, le coup d'État du général Pak Chŏnghŭi amorce le début d'une dictature qui se maintiendra jusqu'à son assassinat, en 1979, et se poursuivra - après un bref intervalle démocratique - pendant toute la présidence du général Chŏn Duhwan (1980-1988) qui lui aussi accédera au pouvoir grâce à un coup d'état. Le général No T’aeu (1988-1993) - dauphin de Chŏn Duhwan - sera élu démocratiquement à la suite d'une révolution étudiante ayant mis à profit, pour renverser la dictature, le fait qu'à l'approche des jeux olympiques de Séoul l'attention de la communauté internationale était braquée sur la Corée du Sud. De ces trois généraux, les deux premiers dirigeront le pays avec une poigne de fer, écrasant toute opposition sans le moindre scrupule en prenant systématiquement pour prétexte la menace d'une agression de la Corée du Nord. L'administration de No T'aeu constitue une étape intermédiaire entre la dictature et l'élection de présidents d'origine non plus militaire mais civile. L'époque allant de 1960 à 1993 est caractérisée par une stabilité politique permettant une croissance économique telle que la Corée du Sud deviendra, avec Hong-kong, Singapour et Taiwan, l'un des «quatre tigres » de l'Asie. Cette stabilité permet donc à la partie sud de la péninsule, dans son ensemble, d'entrer dans une période de prospérité, et au bouddhisme coréen de connaître une véritable renaissance, d'autant plus grande que les trois généraux qui la dominent étaient tous ou

14. Auquel a succédé le culte de son fils Kim Jŏngil, actuel président de la Corée du Nord, qui - au moment où ces lignes sont écrites - n'est plus apparu en public depuis août 2008, époque à laquelle il aurait été frappé d'un accident vasculaire cérébral. 
favorable au bouddhisme (dans le cas de Pak Chŏnghŭi ${ }^{15}$ ) ou bouddhistes euxmêmes dans le cas de ses successeurs. Le succès de cette période sera couronné par l'accession de la Corée du Sud à la démocratie et l'accueil des jeux olympiques de Séoul.

Ce renouveau du bouddhisme se poursuit pendant les présidences de Kim Yŏngsam (1993-1998), un protestant, ainsi que celles de Kim Daejung (19982003 ) et No Muhyŏn (2003-2008), deux catholiques. Il est vrai que les administrations présidentielles sud-coréennes, militaires ou civiles, ont toujours eu tout à gagner à veiller aux intérêts d'une population bouddhiste qui représente quelque $25 \%$ de l'électorat ${ }^{16}$. D'une administration à l'autre, avant ou après la démocratisation, la manière de procéder ne varie guère : distribution avec prodigalité, généralement par le ministère de la culture et du tourisme, d'importants fonds destinés à la restauration, l'entretien, l'embellissement ainsi que l'agrandissement des propriétés monastiques. La réception de ces fonds est d'autant plus attrayante que leur usage n'a jamais été véritablement soumis à un système d'audit crédible. La faible centralisation du schéma organisationnel de l'ordre Chogye ne favorise guère la mise en place d'un système de contrôle plus strict. D'aucuns voient dans ce problème le principal obstacle à une véritable renaissance, c'est-à-dire à une complète réforme du bouddhisme coréen.

\section{Le canon chinois des écritures bouddhiques}

L'une des principales caractéristiques de la renaissance du bouddhisme de Corée du Sud à partir des années soixante a été la traduction en langue coréenne ${ }^{17}$ du canon bouddhique chinois ${ }^{18}$. Cette entreprise, commencée en 1961 et terminée en 2001, aura duré quatre décennies ; elle a été menée par l'Atelier de traduction du canon bouddhique de l'université Tongguk ${ }^{19}$ et s'est achevée avec la publication d'un total de trois cent dix-huit ouvrages ${ }^{20}$, également disponibles sur un

15. Son épouse était une bouddhiste convaincue.

16. $22,8 \%$ selon les résultats du recensement de 2005 .

17. Langue rattachée à la famille ouralo-altaïque et dont la structure est apparentée, en Europe, au basque, au finlandais et au hongrois, ou bien, en Asie, au japonais et au mongol. Notons cependant que plus de $70 \%$ des mots de la langue coréenne courante sont d'origine chinoise ; cette proportion augmente jusqu'à $90 \%$ dans la langue spécialisée. Tandis que la Corée du Nord a supprimé l'apprentissage des caractères chinois, la Corée du Sud maintient l'étude de quelque mille sinogrammes à l'école secondaire.

18. Il s'agit plus précisément du Koryŏ Taejangyŏng, la version de ce canon qui a été gravée, entre 1073 et 1090, des deux côtés de 81258 plaques de bois pesant chacune 3,5 kg; elle est entreposée au monastère Haein, dans le Kyŏngsang du Nord et fait partie du patrimoine culturel de l'humanité. C'est cette version qui a servi de base à la composition de l'Édition Taishō du canon bouddhique sino-japonais.

19. Tongguk Yŏkkyŏngwŏn.

20. Ces ouvrages ont été mis sur le marché au fur et à mesure de leur achèvement. 
disque compact en vente dans le commerce mais dont certains peuvent être obtenus gratuitement sur l'internet ${ }^{21}$. Avant l'apparition de cette traduction, l'ensemble dudit canon n'était accessible qu'aux connaisseurs de la langue chinoise écrite classique, dont l'apprentissage requiert des études spécialisées. Cette situation compliquait singulièrement l'étude du bouddhisme.

Si l'une des raisons permettant d'expliquer la propagation du bouddhisme partout en Asie est sa formidable capacité d'adaptation à différentes cultures, et que la traduction du canon fait partie de la panoplie des moyens dont il s'est servi pour parvenir à cette fin - à la différence de l'hindouisme dont l'enseignement est toujours demeuré lié au sanskrit -, on peut se demander pourquoi les Coréens ont tant tardé à accomplir cette tâche. Un simple coup d'œil aux multiples tribulations que connaissait le bouddhisme du pays du Matin Calme au moment de la promulgation de l'alphabet coréen ${ }^{22}$, en 1446, ainsi qu'à celles qu'il a connues par la suite, suffit pour comprendre que les conditions nécessaires au lancement d'une telle entreprise n'ont pu être réalisées qu'à partir du début des années soixante.

Il est intéressant de noter que, sitôt cette traduction terminée, ses auteurs ont commencé à la reprendre afin de l'améliorer car, reconnaissent-ils, elle comporte beaucoup d'erreurs que l'expérience acquise permet désormais de corriger ${ }^{23}$. L'histoire de la traduction du canon bouddhique indien en chinois comporte elle aussi au moins deux grands moments : le premier avec Kumāraj̄̄iva (343-413), au début du $\mathrm{V}^{\mathrm{e}}$ siècle; le second avec Xuanzang (596-664), dont la nouvelle traduction fut achevée en 645. Il suffit de connaître le volume (quelque cent mille pages) et l'extrême diversité doctrinale de ce canon pour réaliser l'ampleur, la complexité et le coût de cette tâche. En Chine, les ateliers de traduction des textes en provenance d'Inde et d'Asie Centrale pouvaient employer un nombre considérable de personnes et étaient entièrement financés par le pouvoir impérial. En dépit de ces difficultés, l'ordre Chogye ne pouvait plus différer cette entreprise : elle était devenue indispensable pour permettre au bouddhisme sud-coréen de se maintenir sur un pied d'égalité avec le christianisme, son principal concurrent, qui avait commencé à produire des traductions complètes de la Bible longtemps auparavant ${ }^{24}$. Il ne pouvait pas non plus continuer à demeurer à la traîne du bouddhisme du Japon ; celui-ci avait déjà beaucoup plus tôt produit une version en japonais courant de l'Édition Taishō du canon bouddhique sino-japonais.

21. http://www.tripitaka.or.kr

22. Le han'gŭl n'a été créé qu'au XVe siècle par le roi Sejong (1418-1450). Avant sa promulgation par un édit royal, les Coréens utilisaient différentes adaptations du système d'écriture chinois. L'adoption du han'gŭl a été fort lente, à tel point que les lettrés ont continué à se servir des sinogrammes pendant toute l'époque Chosŏn et même sous la colonisation japonaise.

23. Ces erreurs sont à l'origine de la plus ou moins bonne réputation assez vite acquise par cette traduction et expliquent en partie le relatif succès de sa commercialisation.

24. La première traduction complète du Nouveau Testament est parue en 1900 et celle de la Bible en 1910. 
En dépit de la réalisation de cette traduction, la taille même du canon bouddhique - bien que fièrement mise en avant par de nombreux bouddhistes impressionne tant la masse des fidèles que la pensée d'en aborder la lecture ne leur viendra jamais à l'esprit ${ }^{25}$. Il se greffe, de plus, sur cette idée d'un océan de littérature d'une ampleur telle que le lecteur ordinaire ne peut que s'y noyer, la certitude d'un contenu fait d'arcanes dont l'intelligence est au-delà de la portée du commun des mortels. C'est la raison pour laquelle le Bonze Wŏrun, directeur de l'Atelier de traduction de l'université Tongguk, a tenu à préciser - au moment où l'entreprise a été complétée - que $75 \%$ des textes du canon étaient composés de récits et anecdotes dont la compréhension était accessible à tous.

Ces encouragements n'ont cependant pas empêché l'Association pour la promotion du bouddhisme ${ }^{26}$ de mettre au point et de lancer en 2005 l'équivalent d'une "Bible bouddhique » ${ }^{27}$, ou plus exactement, si on en juge à son épaisseur (moins de sept cents pages), d'un " Nouveau Testament bouddhique ». Il s'agit d'un ouvrage entièrement bilingue - l'anglais sur la page de gauche et le coréen sur celle de droite - constitué de l'assemblage, par thèmes couvrant tous les aspects de la vie humaine, d'un certain nombre d'extraits du canon susceptible d'édifier les fidèles. Ce bilinguisme est de toute évidence inspiré par l'existence de nombreuses bibles bilingues; il est d'une importance considérable dans un pays dont l'existence demeure dans l'orbite géostratégique des États-Unis ${ }^{28}$ et qui, en conséquence, accorde la priorité à l'apprentissage de la langue anglaise et non à celle de la Chine et du Japon, ses voisins immédiats. L'ouvrage est mis gratuitement à la disposition de tous, sur le modèle de la Bible de l'Association internationale des Gédéon.

Par-delà l'effort systématique de traduction de l'ensemble du canon ou la création d'une "Bible bouddhique ", certains textes classiques connaissent un destin à part. Tel est le cas du célèbre Sūtra de l'Estrade du VI Patriarche Huineng (638-713): de loin le texte le plus fondamental de toutes les écoles méditatives du monde sinisé. À tel point que c'est souvent celui dont les bonzes proposent la lecture à ceux qui commencent à s'intéresser au bouddhisme. Si quelques-unes des nombreuses versions connues de ce texte ${ }^{29}$ ont été régulièrement traduites en coréen à partir des années soixante, il faut attendre 1987 pour voir apparaître une première traduction en coréen de la version dite de Dunhuang; découverte en 1907, par le Hongrois Aurel Stein (1862-1943), elle est considérée comme la

25. Pour le comprendre, on peut songer au cas de la Bible qui, quoique beaucoup moins volumineuse, demeure un ouvrage difficile à aborder et qui n'est que rarement lue en entier, y compris par les fidèles et les spécialistes.

26. Pulgyo Chŏndo Hyŏphoe.

27. Pulgyo Sŏngjŏn, signifiant littéralement « ouvrage sacré bouddhique ». Le mot sŏngjŏn est également employé pour nommer la Bible.

28. Vingt-huit mille soldats américains demeurent en Corée du Sud.

29. Il existe, en plus du manuscrit de Dunhuang, au moins seize versions dont quatre font plus particulièrement autorité. 
plus ancienne de toutes ${ }^{30}$; c'est cette ancienneté qui, aux yeux de beaucoup, confère au manuscrit de Dunhuang du Sütra de l'Estrade une incomparable autorité. Dès 1967, Philip B. Yampolsky en a publié une édition critique accompagnée d'une traduction en anglais qui continuent à faire autorité. On est donc en droit de se demander pourquoi la Corée du Sud - pays dont la tradition méditative n'a cessé de se réclamer de l'autorité du VI ${ }^{\mathrm{e}}$ Patriarche - a attendu si longtemps pour produire une première traduction de cette version. On peut également s'interroger sur le fait que son auteur soit le Vénérable Sŏngch'ŏl (1912-1993), qui a été maître de méditation du monastère Haein (1967-1993), le plus puissant de Corée du Sud, et patriarche suprême (1981-1993) de l'ordre Chogye.

\section{Le Sūtra de l'Estrade : la traduction de Sŏngch'ŏl}

Selon toute vraisemblance, Sŏngch’ŏl est le premier coréen à avoir pleinement pris conscience du parti qu'il pouvait tirer de la publication d'une traduction annotée de la version de Dunhuang du Sütra de l'Estrade. Cette hypothèse est d'autant plus plausible que cette traduction est le couronnement de toute sa vie et de son œuvre doctrinale. Un examen concis - mais précis - des grandes étapes de son existence ainsi que de la structure de cette œuvre et de son contenu devrait suffire pour le démontrer (Senécal, 2004 : 10-69, 683-713).

Sŏngch'ŏl est né en 1912, sous le nom de I Yŏngju, au tout début de la colonisation de la péninsule par le Japon ; il était le fils aîné d'une famille paysanne aisée de la partie méridionale du Kyŏngsang, dans le Sud-Est coréen. Bien que sa mauvaise santé l'ait empêché de poursuivre ses études au-delà de l'école primaire, il aurait passé son adolescence à lire tous les ouvrages sur lesquels il pouvait mettre la main, y compris métaphysiques, quitte à les troquer contre des sacs de riz. Marié vers l'âge de vingt ans, il se révèle beaucoup plus attiré par la pratique de la méditation que par la vie familiale; il quitte définitivement sa femme et sa fillette quelques années plus tard, en 1936, pour commencer une vie monastique.

I Yŏngju se dirige alors vers le monastère Haein, situé dans le Nord-Kyŏngsang et surnommé «le trésor du Dharma » du bouddhisme coréen ${ }^{31}$; il y est admis par Ha Dongsan (1890-1965), un maître de méditation fort connu à l'époque qui lui donne le prénom monastique «Sŏngch'ŏl ». Sitôt après avoir reçu sa première tonsure, Sŏngch'ŏl quitte le site de Haein et commence à mener la vie d'un bonze se consacrant à la méditation : sans lieu d'attache fixe, résidant dans

30. C'est le volume 2007 du tome 48 de l'Édition Taishō du canon bouddhique sinojaponais, correspondant au manuscrit Stein 5475.

31. Parce que le Koryŏ Taejangyŏng s'y trouve entreposé. 
différents monastères et ermitages au gré des saisons de retraite ${ }^{32}$. Quatre ans plus tard, après avoir consacré tout son temps à la pratique de la méditation et à la lecture du canon bouddhique - il a acquis la réputation de l'avoir appris par cœur et à l'envers -, il obtient, à l'âge de vingt-neuf ans, le bien suprême du bouddhisme : l'Éveil.

Sŏngch'ŏl passe environ vingt-six ans dans la solitude, séjournant dans différents lieux reculés et se consacrant, tour à tour, à la méditation et à l'étude des textes. Cette longue période est cependant interrompue de 1947 à 1949, époque pendant laquelle il participe activement à la vie de l'association dite du monastère Pongam $^{33}$ : un mouvement extrêmement radical de réforme du bouddhisme coréen, préconisant un retour intégral à l'enseignement du Buddha Śākyamuni. Les événements contraignent les membres de l'association à se disperser. La région où se trouve le monastère Pongam est en effet devenue dangereuse, car les troupes gouvernementales y luttent contre la guérilla menée par les communistes. L'année suivante, la guerre de Corée éclate et Sŏngch'ŏl se réfugie dans la poche de résistance tenue par les troupes alliées dans l'extrême sud de la péninsule; il s'y construit un petit ermitage au pied d'une montagne. C'est alors que sa notoriété devient considérable : à tel point qu'il exige de tous ceux et celles qui souhaitent le rencontrer, désormais fort nombreux, l'exécution de trois mille prosternations devant Buddha. De 1956 à 1963, alors que la campagne de purification du bouddhisme coréen ordonnée par Sungman Rhee bat son plein, Sŏngch'ŏl refuse toutes les nominations qui lui sont proposées par les autorités monastiques. Pour trouver la paix, il se réfugie dans un ermitage de montagne, difficile d'accès, mais qu'il fait néanmoins entourer d'une clôture de fil de fer barbelé. Il aurait consacré toutes les années passées en ce lieu, assis en méditation, et sans jamais se coucher. En 1966, six ans après le coup d'état de Pak Chŏnghūi, Sŏngch'ŏl sort de la solitude et commence ce qui peut être appelé sa vie publique ; il a cinquante-six ans.

En 1967, Sŏngch’ŏl est nommé maître de méditation du monastère Haein, un poste qu'il accepte et dans lequel il demeurera jusqu'à sa mort. C'est au cours de cette même année qu'il prononce, à la faveur de la retraite estivale, la prédication dite « des Cent Soleils de la Porte du Dharma ${ }^{34}$ » : un enseignement étalé sur trois mois, au rythme de deux heures par jour, dans lequel il propose une vaste synthèse de tout le bouddhisme, entièrement centrée sur la doctrine de la voie moyenne ${ }^{35}$. Cet enseignement va jusqu'à remettre complètement en

32. Ces retraites sont au nombre de deux par an, l'une pendant l'été et l'autre l'hiver, et durent trois mois chacune.

33. Pongamsaŭi Kyŏlsa.

34. $A b$. "Prédication des Cent Soleils » dans la suite de cet article.

35. Le texte intégral en a été publié en mars 1992 sous la forme d'un volume en deux tomes totalisant sept cents pages également disponible sur disque compact. Sŏnch'ŏl s'est inspiré de semblables tentatives de synthèse réalisées au Japon. 
cause celui de Chinul (1158-1210), alias Puril Pojo (Buddha-Soleil rayonnant dans toutes les directions). Ce dernier est à fois le fondateur du monastère Songgwang, surnommé "le trésor du Sangha » du bouddhisme coréen, dans le Sud-Ouest de la péninsule, et le plus grand maître national de méditation de toute l'époque Koryŏ. L'autorité incontestée de Chinul avait jusqu'alors dominé tout le bouddhisme péninsulaire. Cette remise en cause de huit siècles d'histoire doctrinale fait l'effet d'une bombe jetée au milieu du sangha. C'est la raison pour laquelle il n'est pas exagéré de dire que la prédication des Cent Soleils équivaut à l'exécution d'un véritable coup d'état «doctrinal» au sein du bouddhisme coréen. C'est d'autant plus vrai que le monastère Songgwang et la région où il se trouve, le Chŏlla, constituent les rivaux traditionnels du monastère Haein et du Kyŏngsang. Rien d'étonnant alors à ce que Sŏngch'ŏl ait aussitôt été accusé de prêcher une doctrine sortie de l'orbite de l'orthodoxie de la tradition méditative coréenne ; pour se défendre et démontrer que ce n'est pas lui mais Chinul et ses adeptes qui ne sont pas orthodoxes, il commence à rédiger son œuvre doctrinale. Au cours des années suivantes, avec la parution de chaque nouvel ouvrage, la tension entre lui et ses adversaires ne cesse de croître; elle deviendra ce que l'on appelle " la querelle subito-gradualiste coréenne ». Le combat doctrinal de Sŏngch'ŏl se terminera en 1987, avec la publication de La Traduction annotée de la version de Dunhuang du Sūtra de l'Estrade ; pour être correctement compris, cet ouvrage doit être entendu comme le dernier cri de son auteur. Entre-temps, moins d'un an après le coup d'état ayant porté au pouvoir le général Chŏn Duhwan, et alors que le bouddhisme coréen demeure profondément traumatisé par les conséquences des descentes de police effectuées sur les monastères à l'automne précédent, Sŏngch'ŏl est nommé patriarche suprême de l'ordre Chogye, poste qu'il occupera jusqu'à sa mort en 1993.

Pendant toutes les années de sa vie publique, Sŏngch'ŏl ne quittera pratiquement jamais le monastère Haein. Il réside plus précisément à l'ermitage Paegnyŏn (du Lotus Blanc), situé plus haut dans la montagne, à une distance d'un peu moins d'un kilomètre de l'enceinte principale du site. Lorsque Pak Chŏnghŭi visite Haein en 1978, Sŏngch'ŏl ne daigne pas descendre de son perchoir pour aller l'accueillir. En 1984, il refuse de se rendre à Séoul pour rencontrer JeanPaul II qui avait exprimé le désir de faire sa connaissance. L'aspiration constante de Sŏngch'ŏl à la solitude, son langage tenant de l'idiolecte, ses tendances excentriques et misanthropiques prononcées, ainsi que la férocité de son caractère refusant tout compromis lui vaudront le surnom de Tigre des monts Kaya ${ }^{36}$, du nom du massif montagneux au sein duquel se trouve le monastère Haein.

Lorsque Sŏngch'ŏl meurt en 1993, la Corée du Sud toute entière s'arrête pour célébrer ses obsèques. Pendant quarante-huit heures, l'événement accapare

36. Du nom de la ville indienne de Gaya à proximité de laquelle le Buddha obtint l'Éveil. 
complètement l'attention des médias. Entre le moment de sa mort et le quaranteneuvième jour y faisant suite, plus de deux millions de visiteurs se présenteront au monastère Haein, soit pour honorer sa dépouille ou assister à ses funérailles, soit pour pouvoir jeter un coup d'œil aux reliques de ses cendres. Celles-ci seront ensuite déposées dans un magnifique stūpa érigé au milieu d'une esplanade, le tout construit en marbre importé de l'Inde et situé bien en vue à l'entrée du monastère Haein. Si Sŏngch’ŏl a été perçu par les masses comme un grand réformateur du bouddhisme coréen, de la dimension de Huineng ou du Buddha Śakyamuni, il est aussi entré dans l'histoire comme un personnage fortement controversé par nombre de bonzes et d'universitaires.

L'œuvre doctrinale de Sŏngch'ŏl se compose de deux parties principales, respectivement intitulées: Recueil des propos sur le Dharma du Vénérable Sŏngch'ŏl et La Collection des anciens miroirs de l'école Sŏn. Tel que son nom l'indique, la première, qui compte onze volumes, est un recueil de tous les enseignements de l'auteur; elle se subdivise en deux autres : celle des ouvrages qu'il a rédigés avec l'aide de ses disciples - au nombre de sept - puis celle de ceux entièrement rédigés de sa main, de loin la plus importante, qui en compte quatre. Ces ouvrages sont, dans l'ordre : La Traduction annotée du manuscrit de Dunhuang du Sūtra de l'Estrade (1987) ; La Voie correcte du Sŏn (1981), Magnificence de l'origine (1982) et La Lignée de transmission du Dharma au sein $d u$ bouddhisme coréeen (1976). Il est remarquable que la traduction du Sütra de l'Estrade occupe la première place, en dépit de l'année de sa publication. La seconde partie de l'œuvre de Sŏngch'ŏl se compose de la traduction - du chinois classique en coréen - de trente-sept ouvrages dont l'auteur juge la connaissance indispensable à la pratique de la méditation ${ }^{37}$; une introduction présente chaque volume. Il s'agit donc, d'abord et avant tout, d'une œuvre de traduction. Il va sans dire que ces deux grandes parties de l'œuvre de Sŏngch'ŏl forment un tout fortement cohérent où tous les éléments se renvoient les uns aux autres dans un but précis : démontrer que Chinul s'est trompé et que l'heure de rétablir la véritable orthodoxie de l'école méditative a sonné.

Quelle est donc cette orthodoxie ? En réalité, par delà sa complexité, l'enseignement de Sŏngch'ŏl peut être ramené à quelques éléments simples : le bouddhisme est la religion de l'Éveil ; on peut l'atteindre en méditant la phrase ou le mot clef ( $h w a d u)$ de ces énigmes insolubles pour la raison que sont les kōans ${ }^{38}$; l'Éveil est atteint non pas progressivement mais subitement ; la pratique de celui qui a véritablement atteint l'Éveil équivaut à celle d'un Buddha vivant, tout retour en arrière est impossible. Chinul - tout en ayant lui aussi reconnu à la fin de sa vie la supériorité de cette position qualifiée de "subito-subitiste ${ }^{39}$ »-

37. Ces ouvrages ne faisant pas partie du canon bouddhique mais de celui de l'école méditative, ils nécessitent une traduction à part.

38. Cette pratique s'appelle le kanhwasŏn.

39. Tono tonsu. 
a cependant continué à accepter tout autant la possibilité d'une expérience dite "subito-gradualiste ${ }^{40}$ »: un Éveil devant être suivi pendant fort longtemps, sinon indéfiniment, d'une pratique progressive. C'est précisément la recherche d'un équilibre entre ces deux paradigmes, servant à définir l'Éveil, que dénonce Sŏngch'ŏl; il accuse Chinul de faire un compromis, qui ne peut mener nulle part, entre l'enseignement des écoles scolastiques, subito-gradualiste, et celui des écoles méditatives, subito-subitiste. Ce faisant, Sŏngch'ŏl se pose comme le seul véritable maître de méditation capable de rétablir l'orthodoxie perdue depuis huit cents ans; il en tient pour preuve ultime la version la plus ancienne de l'enseignement du $\mathrm{VI}^{\mathrm{e}}$ Patriarche.

En réalité, cette querelle doctrinale est dans sa majeure partie tout au moins une réédition coréenne d'un vieux conflit entre les écoles scolastique et méditative de Chine dont la source lointaine remonte aux origines du bouddhisme dans l'antiquité indienne. L'étude de la tension historique entre ces deux conceptions opposées de l'Éveil déborde la visée de cet article. Examinons donc deux points relatifs à l'enseignement de Sŏngch'ŏl dans le contexte coréen : la validité de l'accusation qu'il formule contre Chinul ; l'usage qu'il fait de la plus ancienne version du Sütra de l'Estrade.

Une étude attentive de l'œuvre de Sŏngch'ŏl révèle qu'il n'a jamais cherché à comprendre Chinul en le resituant dans le contexte historique qui lui est propre. La tâche de ce dernier consistait, au XII siècle, à réconcilier les écoles scolastique et méditative qui ne cessaient de s'entre-déchirer au sein du royaume de Koryŏ, en dépit des tentatives successives menées par le pouvoir royal pour les pacifier et les unifier. De cela, Sŏngch'ŏl n'a cure. Tout se passe comme si, plutôt que de s'intéresser vraiment à Chinul, il s'en servait comme d'un adversaire dont l'attaque constante fondait sa raison d'être et justifiait l'exposé toujours plus précis de sa doctrine.

Quant à la version de Dunhuang du Sūtra de l'Estrade, la méthode historicocritique permet effectivement de conclure qu'elle est la plus ancienne connue à ce jour ; mais ladite méthode ne permet pas pour autant d'affirmer que cette version véhicule une doctrine de l'Éveil strictement subitiste, au détriment de tout gradualisme, et que son auteur serait le $\mathrm{VI}^{\mathrm{e}}$ Patriarche en personne. En réalité, on ne sait pratiquement rien du dénommé Huineng et les meilleures exégèses de la version de Dunhuang révèlent un processus de composition complexe faisant intervenir non pas une seule mais plusieurs couches rédactionnelles, rattachées à plusieurs époques et représentant diverses tendances doctrinales ${ }^{41}$. En outre, de nombreux autres documents de Dunhuang révèlent qu'une opposition trop stricte du subitisme et du gradualisme tient plus de la caricature que de la réalité de ce qui se passait alors en Chine sous la dynastie des Tang (618-907).

40. Tono chŏmsu.

41. Chŏng, 2007 : 81-83. 
On peut ajouter que le mauvais état du manuscrit de Dunhuang rend fortement aléatoire l'interprétation exacte de certains passages. Tout cela permet de comprendre que Chinul, bien qu'il ait accordé au moins autant d'importance au Sütra de l'Estrade que son détracteur du $\mathrm{XX}$ siècle, au point d'en faire l'un des trois piliers de sa pensée, en ait tiré des conclusions fort différentes (Chŏng, 2007 : 81-83). Mais ces apports de la méthode historico-critique n'ont jamais vraiment intéressé Sŏngch’ŏl.

Jusqu'au bout, Sŏngch’ŏl a tenu à voir dans la version de Dunhuang, non seulement les ipsissima verba du grand Huineng, mais encore les paroles du Buddha Śakyamuni en personne. C'est fort de cette certitude qu'il proclame de plus en plus, à temps et à contretemps, le message qui se résume ainsi : "Chinul et ses adeptes se sont trompés! C'est moi qui ai raison! Si vous n'en êtes pas parfaitement convaincus, vous n'avez qu'à lire la version de Dunhuang du Sūtra de l'Estrade ». Sachant que Sŏngch'ŏl est d'abord et avant tout un autodidacte qui n'a jamais été formé aux exigences de la méthode historico-critique, on aurait tort de trop lui tenir rigueur de ces excès. Les nombreux universitaires coréens spécialisés dans l'étude de la doctrine de Chinul n'ont cependant pas pris cette accusation à la légère. C'est la raison pour laquelle ils ont fondé, dès 1988, le Pojo Sasang Yŏnguwŏn, un institut de recherche très dynamique qui publie une excellente revue universitaire sur la pensée de Chinul. Les partisans de Sŏngch'ŏl ont répliqué en fondant un centre de recherche sur la doctrine de leur propre maître.

Un passage précis de la version de Dunhuang du Sūtra de l'Estrade, citant les paroles d'une stance qui aurait été formulée par Huineng, permet de penser que Sŏngch'ŏl a fini par s'identifier complètement au VI ${ }^{e}$ Patriarche : « Ne transmettez que l'enseignement subitiste ; allez dans le monde détruire les doctrines erronées » ${ }^{42}$. Ce faisant, Sŏngch'ŏl ne tient pas compte d'autres passages qui parlent du gradualisme sur un mode inclusif, tel par exemple : «Depuis les temps anciens, le subitisme et le gradualisme de la doctrine que je vous propose font tous deux du "sans-penser" leur point cardinal " ${ }^{43}$. En affirmant que les deux sinogrammes correspondant au subitisme et au gradualisme seraient erronés, il traduit par : "Depuis les temps anciens, dans son entier la doctrine que je vous propose fait du "sans-penser" son point cardinal " ${ }^{44}$. Bien que cette option puisse mériter considération, il est significatif que l'ensemble des autres traducteurs coréens de la version de Dunhuang du Sütra de l'Estrade aient choisi de l'ignorer.

À ce jour, il s'est vendu quelque vingt-cinq mille exemplaires de La Traduction annotée du manuscrit de Dunhuang du Sütra de l'Estrade, ce qui est beaucoup dans le monde de l'édition bouddhiste, d'autant plus que l'ouvrage continue à se

\footnotetext{
42. 2007 , vol. 48 , p. 341 c 18 .

43. Id. p. 338 c 3 .

44. I, $1987: 54$
} 
vendre. Qu'on soit d'accord avec son auteur ou non, il faut reconnaître qu'un puissant souffle la traverse tout entière et que, même si elle ne tient pas compte des conclusions de la méthode historico-critique, elle fait preuve d'une cohérence interne qui lui confère une grande puissance de fascination. Si on en juge au fait que la simple déclaration d'un intérêt personnel pour le Sūtra de l'Estrade suscite souvent la question : "Quelle version lisez-vous ? ", on ne peut douter que la traduction de Sŏngch'ŏl ait eu un réel impact sur l'ensemble du bouddhisme coréen.

Avant 1987 d'une façon générale, le bouddhisme de Corée du Sud n'attachait pas d'importance spéciale à la version de Dunhuang, d'autant plus qu'on estimait connaître suffisamment Huineng et son enseignement grâce aux autres versions du Sūtra de l'Estrade, tout particulièrement le manuscrit de Deyi ${ }^{45}$. En faisant de La Traduction annotée du manuscrit de Dunhuang du Sūtra de l'Estrade le couronnement de toute sa vie et son œuvre, le patriarche suprême de l'ordre Chogye a complètement bouleversé cette situation. À tel point qu'il n'est pas exagéré de voir dans cette traduction l'équivalent d'une ligne de partage des eaux dans l'histoire du bouddhisme coréen : entre les partisans d'un strict retour aux fondements de la tradition méditation extrême-orientale - tels qu'ils seraient consignées dans ce manuscrit - et les défenseurs de la nécessité de se tourner radicalement vers l'avenir. C'est la raison pour laquelle on assiste, après 1987 , à l'apparition de nouvelles traductions de la version de Dunhuang et à un regain d'intérêt pour la figure du VI ${ }^{\mathrm{e}}$ Patriarche.

\section{Essai de classification des traductions}

Outre la publication de plusieurs études générales sur Huineng et le Sūtra de l'Estrade, cinq traductions sérieuses du manuscrit de Dunhuang ont été publiées après celle de Sŏngch'ŏl. Il faut cependant attendre 1992 pour voir apparaître la première ; et encore, celle-ci n'est que la traduction en coréen de l'édition critique de Yampolsky qui, soit dit en passant, a manifestement influencé toutes les autres bien que n'étant jamais citée. Entre temps, on peut signaler la publication, dès 1988, d'une excellente bande dessinée illustrant le Sūtra de l'Estrade, mais sans référence particulière au manuscrit de Dunhuang. La parution des quatre autres traductions de celui-ci s'est effectuée au cours des dernières années : trois en 2003 et une en 2008 ; elles sont toutes accompagnées de commentaires et de notes. Ce long délai s'explique par le temps requis pour mesurer l'impact de la traduction de Sŏngch'ŏl et réaliser la nécessité d'y répondre, ainsi que celui nécessaire pour bien traduire. Signalons encore la publication, en 2007, d'une étude très fouillée, exclusivement consacrée à la version de Dunhuang du Sūtra de l'Estrade. Les quatre traductions coréennes peuvent être classées en trois types distincts.

45. Tome 48, 2008. 
Le premier correspond à la méthode historico-critique. Son représentant est Chŏng Sŏngbon, un bonze enseignant le bouddhisme à l'université Tongguk ; formé au Japon, il passe pour le meilleur spécialiste de la tradition méditative coréenne. Ce dernier ne cache en rien son aversion pour le subitisme et sa préférence pour l'approche gradualiste de l'Éveil. Il n'épargne aucun effort pour démythifier tant le manuscrit de Dunhuang du Sūtra de l'Estrade que la figure du $\mathrm{VI}^{\mathrm{e}}$ Patriarche. Ses critiques, dont les adeptes de Sŏngch'ŏl, lui reprochent de n'être qu'un intellectuel qui n'a jamais vraiment pratiqué la méditation et qui, en conséquence, n'y comprend strictement rien. Il est vrai que les commentaires de Chŏng Sŏngbon fournissent une quantité d'informations telle qu'elle finit par noyer complètement le lecteur.

Le deuxième type correspond à la traduction de Maître Ch’ŏnghwa (19142003), un bonze spécialiste de la méditation dans l'esprit de l'école de la Terre Pure. Tout en ressemblant étrangement dans son ensemble à celle de Sŏngch'ŏl, la traduction de Ch'ŏnghwa s'en distingue néanmoins clairement sur deux points précis : il reconnaît tout autant le subitisme que le gradualisme; il rejette la pratique des kōans - fer de lance de l'approche "sŏngch'ŏllienne » - au profit de celle de la commémoration de Buddha dans la ligne de l'amidisme. Mais quoiqu'en dise Sŏngch'ŏl, il n'est jamais question de kōans dans le manuscrit de Dunhuang; pas plus d'ailleurs que de commémoration de Buddha, sinon pour la dénoncer. Il est clair que Ch'ŏnghwa, contemporain et rival de Sŏngch'ŏl, a repris à son profit l'inspiration de ce dernier, afin de mettre lui aussi son enseignement sous l'autorité du VI ${ }^{\mathrm{e}}$ Patriarche.

Le troisième type de traduction a été produit par Kim Yunsu et Chŏn Chongsik, deux laïcs, ni professeurs d'université ni spécialistes de la méditation, cherchant tout simplement à mettre le bouddhisme en pratique au cœur de la cité. Ni l'un ni l'autre ne paraît indûment attaché au subitisme. Dans un contexte tel qu'un nombre croissant de laïcs cherchent à s'affirmer et à s'organiser afin de s'affranchir de l'autorité de l'ordre Chogye, ces traductions sont d'autant plus significatives qu'elles remettent en cause le monopole de l'interprétation de l'enseignement du VI Patriarche exercé par le monde monastique.

Aucun des commentaires accompagnant ces trois traductions ne fait explicitement référence à la traduction de Sŏngch'ŏl, bien qu'ils y répondent manifestement tous. La raison en est que ce dernier, bien que hautement controversé, demeure profondément respecté par l'ensemble du bouddhisme coréen. Tout se passe néanmoins comme si, après le ton absolu d'une traduction annotée qui a cherché à s'imposer comme définitive en 1987, un concert à plusieurs voix s'était amorcé. 


\section{La fin de la tour de Babel}

Si la traduction du manuscrit de Dunhuang du Sūtra de l'Estrade publiée par Sŏngch'ŏl en 1987 se voulait d'abord, à l'instar de toute son œuvre, le couronnement d'une vie toute entière consacrée à la réforme du bouddhisme coréen, elle n'en a pas moins été perçue par beaucoup comme la récupération de l'autorité du VI ${ }^{\mathrm{e}}$ Patriarche Huineng au profit d'une tentative de prise de pouvoir et d'hégémonie par le monastère Haein. La rhétorique de l'immédiateté de l'Éveil mise en avant par Sŏngch'ŏl, quoi qu'il puisse paraître, ne transcende pas complètement les vicissitudes de l'histoire. Autrement dit, Sŏngch'ŏl n'est pas du tout un météore apparu soudainement au sein du bouddhisme coréen ; au contraire, son avènement s'inscrit dans des coordonnées précises. Bien que personne n'ose l'affirmer clairement en Corée du Sud, l'opposition entre subitisme et gradualisme qui traverse toute la pensée "sŏngch'ŏllienne » - avec une telle intransigeance qu'elle a divisé le bouddhisme du pays - évoque l'antagonisme politique qui a servi à justifier depuis soixante ans la division de la péninsule du Matin Calme et qui n'est qu'une actualisation régionale des conséquences de la guerre froide. Ce n'est pas un hasard si la durée de la vie publique de Sŏngch'ŏl correspond pratiquement à celle de la dictature militaire sud-coréenne ; il est d'ailleurs remarquable que ce dernier arrête ni plus ni moins ses activités alors que celleci prend fin en 1988, tout en gardant néanmoins ses titres de maître de méditation du monastère Haein et patriarche suprême de l'ordre Chogye.

Si Sŏngch'ŏl continue à fasciner les masses, c'est parce que sa vie permet de penser qu'un autre monde, le Nirvāṇa, est possible au-delà de celui de l'océan des douleurs, le samsāra, qui constitue le lot du commun des mortels. Au cœur d'un monde complexe, déchiré par les luttes pour la survie et le pouvoir, Sŏngch'ŏl se manifeste comme la figure de l'incorruptible Éveillé proposant un message de salut clair et simple, résumé dans le manuscrit de Dunhuang du Sūtra de l'Estrade. Son existence érémitique dans les hauteurs des monts Kaya, à la limite de l'inaccessible, a fait de lui un être à part sur lequel chacun est libre de projeter le meilleur de ses attentes et espérances. Les médias se sont d'autant plus intéressés à Sŏngch'ŏl qu'il les abhorrait et que certains membres de son entourage ont discrètement veillé à une soigneuse mise en scène de toute son existence. À l'instar des tigres disparus de la péninsule depuis la guerre de Corée, le Tigre des monts Kaya a fini par devenir une créature mythique.

Et pourtant, Sŏngch'ŏl a été d'autant plus près du pouvoir politique qu'il s'en est tenu éloigné. La constance de son silence sous la dictature, alors même qu'il était patriarche suprême de l'ordre Chogye, ne peut qu'interroger ; a fortiori quand on sait que nombreux sont les bonzes qui ont fini par en devenir les victimes. Alors que Chŏn Duhwan faisait effectuer des descentes de police sur tout le monachisme, en 1980, Sŏngch'ŏl s'apprêtait à publier ses principales œuvres sur la pratique de la méditation en vue d'obtenir l'Éveil. Plus troublant encore est le fait qu'il ait laissé sans mot dire, de 1986 à 1990, le «bonzegangster » Sŏ Ŭihyŏn régner en roi et maître à la tête de l'administration de 
l'ordre Chogye ; à tel point que l'expulsion de ce dernier du quartier général de l'ordre, au centre ville de Séoul, aura nécessité l'intervention armée - et entièrement médiatisée - d'une partie du sanghha. On sait que Sŏngch'ŏl avait interdit l'entretien des bâtiments de l'ermitage du Lotus Blanc; de telle sorte que peu de temps avant sa mort, en 1993, l'un d'eux dont la poutre maîtresse avait fini par pourrir s'est effondré. On peut y voir le symbole de la fin d'un monde.

En dépit de l'amorce d'une renaissance à partir de 1953 et de la réforme lancée par Sŏngch'ŏl, le bouddhisme coréen continue à souffrir du discrédit causé par les diverses raisons historiques évoquées dans cet article. L'important soutien financier qui lui a été octroyé par les gouvernements qui se sont succédés depuis cette époque ne supprime en rien les défis auxquels il doit faire face : effectifs stagnants et implantation sociale marginale par rapport au christianisme ${ }^{46}$; faible pouvoir d'attraction sur la jeunesse beaucoup plus facilement attirée par les nouveaux mouvements religieux apparentés au New Age ${ }^{47}$; diminution vritique du nombre de jeunes gens choisissant la vie monastique. Cette situation est source d'une réelle inquiétude quant à l'avenir du bouddhisme coréen. Afin d'y faire face, il y a quelques années, l'ordre Chogye a fait passer l'âge limite de l'admission des nouvelles recrues de quarante à cinquante ans. Le sentiment de crise qui frappe l'ordre Chogye a été aggravé par la nomination à la présidence, en février 2008, de I Myongbak, un évangéliste militant dont l'administration n'a rien fait bien au contraire pour cacher sa profonde aversion à l'égard des bouddhistes. La situation est tendue au point que l'esplanade du quartier général de l'ordre Chogye a été recouverte de lanternes en papier de couleurs variées sur lesquelles le tout venant peut lire les lettres O, U, T (Senécal, 2007 : 54).

C'est sur ce fond de tableau que sont publiées depuis 1992 des traductions de la version de Dunhuang du Sūtra de l'Estrade autres que celle de Sŏngch'ŏl. Elles témoignent de l'existence au sein du bouddhisme coréen d'un puissant mouvement de rejet de la tour de Babel édifiée par ce dernier sous la dictature (Senécal, 2008 : 53). L'avenir dira si la quête d'une approche dialogique de la vérité, dont ce mouvement paraît être le reflet - en conformité avec l'esprit de la démocratie mise en place en 1988 -, permettra ou non au bouddhisme sud-coréen de sortir de l'impasse dans laquelle il se trouve aujourd'hui et d'aller au bout du renouveau amorcé au début des années soixante.

Bernard SENÉCAL

Université Sogang, Département des religions - Séoul senecber@sogang.ac.kr

46. Toutes confessions confondues, le christianisme représente $28 \%$ de la population sudcoréenne. L'engagement social des bouddhistes de Corée du Sud, toutes activités comprises, représente moins de $5 \%$ de celui des chrétiens.

47. À titre d'exemple, moins de deux cents étudiants, sur treize mille, fréquentent assidûment l'aumônerie de l'université Tongguk. 


\section{Bibliographie}

Carré Patrick (trad.), 1995, Le Soûtra de l'Estrade du Sixième Patriarche Houei-neng, Paris, Seuil, "Points- Inédit Sagesses ».

CHŎN Chongsik, 2008, Tonhwangbon Yukcho tan'gyŏng [La Version de Dunhuang du Sūtra de l'Estrade], Séoul, Kyŏngsŏwŏn.

CHŎNG Sŏngbon, 2003, Tonhwangbon Yukcho tan'gyŏng [La Version de Dunhuang du Sūtra de l'Estrade], Séoul, Han'guk Sŏn Munhwa Yŏnguwŏn.

CHŎNG Yujin, 2007, Tonhwangbon Yukcho tan'gyŏng yŏngu [Recherches sur la version de Dunhuang du Sütra de l'Estrade], Séoul, Kyŏngsŏwŏn.

Hitchens Christopher, 2007, God is not Great, New York, Twelve-Hachette Book Group. I SŎNGCH'ŎL, 1976-1993, Sŏngch'ŏl sūnim pŏb' ŏjip, [Recueil des propos sur le Dharma du Vénérable Sŏngch'ŏl], Onze volumes publiés dont, en 1987, La Traduction annotée de la version de Dunhuang du Sūtra de l'Estrade, Haein-sa (Paegnyŏn-am), Changgyŏnggak.

I SŏNGCH'ŎL, (éd.), 1987-1993, Kogyŏng sŏllim ch'ongsŏ [La Collection des anciens miroirs de l'école Sŏn], Trente-sept volumes de différents auteurs traduits, Haein-sa (Paegnyŏn-am), Changgyŏnggak.

KIM Yunsu, 2003, Yukcho tan'gyŏng ilkki [Lecture du Sūtra de l'Estrade], Séoul, Magobooks.

Morel Françoise, (trad.), 2001, Le Soutra de l'Estrade du don de la loi, Paris, La Table Ronde.

Mu Ch’ŏnghwa, 2003, Séoul, Yukcho tan'gyŏng [Le Sütra de l'Estrade], Kwangryun Ch'ulp'ansa.

SenÉCAL Bernard, 2004, "La vie et l'œuvre du Maître Sŏn T’oeong Sŏngch’ŏl (19121993 ) ", thèse de doctorat soutenue à l'université Paris 7 - Denis Diderot, UFR-LCAO.

-, 2007, «A Study for the Understanding of T'oeong Sŏngch'ŏl: How should he be introduced to the Western World? ", Pulgyohak Yŏngu [Études sur le bouddhisme], 17, pp. 33-54.

-, 2008, "Looking for the $1^{\text {st }}$ Step toward Reconciliation between Religions ", Pulgyo Pyŏngnon [Buddhist Review], 37, pp. 53-67.

Takakusu \& Watanabe, (éd.), 1924-1932, Édition Taishō du canon bouddhique sinojaponais (cd-rom), Taipei, Chinese Buddhist Electronic Text Association.

Toulsaly Catherine, (trad.), 1992, Sûtra de la plate-forme, Paris, Librairie You Feng.

Yampolsky Philip B., 1967, The Platform Sutra of the Sixth Patriarch, the Text of the TunHuang Manuscript, Translated, with Notes, New York, Columbia University Press.

\section{Résumé}

En 1987, le Vénérable Sŏngch’ŏl, Patriarche de l'ordre Chogye, de loin la plus puissante organisation monastique du bouddhisme du pays du Matin Calme, publiait la première traduction en coréen du manuscrit de Dunbuang du Sūtra de l'Estrade. Cet événement précède de peu les jeux olympiques de Séoul et la transition du pays d'une dictature militaire à un régime démocratique; il a suscité la production de plusieurs autres traductions du même manuscrit, dont les contenus vont du quasi plagiat de Sŏngch'ŏl à la complète remise en cause des options doctrinales de ce dernier. Ce phénomène s'inscrit dans un contexte formé par une constellation de données historiques, sociopolitiques, économiques, religieuses etc., dont l'étude procure un tableau 
multidimensionnel de l'état et de la situation dans laquelle se trouve le bouddhisme de la république de Corée du Sud, laquelle a célébré en 2008 le soixantième anniversaire de sa fondation.

Mots-clés: Corée, bouddhisme, Sūtra de l'Estrade, version de Dunhuang, traduction.

\section{Abstract}

In 1987, the Venerable Sŏngch'ŏl, Patriarch of the Chogye Order, by far the most powerful monastic organization of South Korean Buddhism, published the first Korean translation of the Dunhuang manuscript of the Platform Sütra. This event shortly preceded the Seoul Olympic Games and the transition of the country from a dictatorship to a democratic regime; it has caused the production of several other translations of the same manuscript, the content of which varies from the quasiplagiarism of Sŏngch'ŏl to the complete challenging of his doctrinal options. This phenomenon is part of a context formed by a constellation of historical, socio-political, economic, religious data etc, the study of which provides a multidimensional picture of the state of Korean Buddhism and the situation it is in as the republic of South Korea has just celebrated, in 2008, the sixtieth anniversary of its foundation.

Key words: Korea, Buddhism, Platform Sutra, Dunhuang manuscript, translation.

\section{Resumen}

En 1987, el Venerable Sŏngch’ŏl, patiarca de la orden Chogya, ciertamente la más potente organización monástica del budismo del país de la Mañana Calma, publicaba la primera traducción en coreano del manuscrito de Dunhuang del Sütra del Estrado. Este acontecimiento, que precedió por poco tiempo a los Juegos Olimpicos de Seul y a la transición del país de una dictadura militar a un régimen democrático; suscitó la producción de muchas otras traducciones del mismo manuscrito, cuyos contenidos van desde el cuasi plagio de Sŏngch'ŏl a al cuestionamiento total de las opciones doctrinales de este último. Este fenómeno se inscribe en un un contexto formado por una constelación de datos históricos, sociopoliticos, económicos, religiosos, etc.; este estudio intenta trazar un cuadro multidimencional del estado de la situación en el que se encuentra el budismo de la república de Corea del Sur, que celebró, en 2008, el $60^{\circ}$ aniversario de su fundación. (trad. Véronica Giménez Béliveau)

Palabras clave: Corea, budismo, Sûtra del Estrado, versión de Dunbuang, traducción. 